\title{
An Atomic-Array Optical Clock with Single-Atom Readout
}

\author{
Ivaylo S. Madjarov, ${ }^{1}$ Alexandre Cooper, ${ }^{1}$ Adam L. Shaw $\odot,{ }^{1}$ Jacob P. Covey, ${ }^{1}$ Vladimir Schkolnik $\odot,{ }^{2}$ \\ Tai Hyun Yoon $\odot,{ }^{1, \dagger}$ Jason R. Williams $\oplus^{2}$ and Manuel Endres $\oplus^{1, *}$ \\ ${ }^{1}$ Division of Physics, Mathematics and Astronomy, California Institute of Technology, \\ Pasadena, CA 91125, USA \\ ${ }^{2}$ Jet Propulsion Laboratory, California Institute of Technology, Pasadena, CA 91109, USA
}

(Received 4 September 2019; revised manuscript received 23 October 2019; published 11 December 2019)

Currently, the most accurate and stable clocks use optical interrogation of either a single ion or an ensemble of neutral atoms confined in an optical lattice. Here, we demonstrate a new optical clock system based on an array of individually trapped neutral atoms with single-atom readout, merging many of the benefits of ion and lattice clocks as well as creating a bridge to recently developed techniques in quantum simulation and computing with neutral atoms. We evaluate single-site-resolved frequency shifts and shortterm stability via self-comparison. Atom-by-atom feedback control enables direct experimental estimation of laser noise contributions. Results agree well with an ab initio Monte Carlo simulation that incorporates finite temperature, projective readout, laser noise, and feedback dynamics. Our approach, based on a tweezer array, also suppresses interaction shifts while retaining a short dead time, all in a comparatively simple experimental setup suited for transportable operation. These results establish the foundations for a third optical clock platform and provide a novel starting point for entanglement-enhanced metrology, quantum clock networks, and applications in quantum computing and communication with individual neutral atoms that require optical-clock-state control.

DOI: 10.1103/PhysRevX.9.041052

\section{INTRODUCTION}

Optical clocks-based on interrogation of ultranarrow optical transitions in ions or neutral atoms-have surpassed traditional microwave clocks in both relative frequency stability and accuracy [1-4]. They enable new experiments for geodesy [2,5], fundamental physics [6,7], and quantum many-body physics [8], in addition to a prospective redefinition of the SI second [9]. In parallel, single-atom detection and control techniques have propelled quantum simulation and computing applications based on trapped atomic arrays; in particular, ion traps [10], optical lattices [11], and optical tweezers $[12,13]$. Integrating such techniques into an optical clock would provide atom-by-atom error evaluation, feedback, and thermometry [14]; facilitate quantum metrology applications, such as quantumenhanced clocks [15-18] and clock networks [19]; and

\footnotetext{
*mendres@caltech.edu

${ }^{\dagger}$ Present address: Department of Physics, Korea University, Seoul 02841, Republic of Korea.

Published by the American Physical Society under the terms of the Creative Commons Attribution 4.0 International license. Further distribution of this work must maintain attribution to the author(s) and the published article's title, journal citation, and DOI.
}

Subject Areas: Atomic and Molecular Physics, Optics, Quantum Physics enable novel quantum computation, simulation, and communication architectures that require optical-clock-state control combined with single-atom trapping [20-22].

As for current optical clock platforms, ion clocks already incorporate single-particle detection and control [23], but they typically operate with only a single ion. Research towards multi-ion clocks is ongoing [24]. Conversely, optical lattice clocks (OLCs) $[1,2,4]$ interrogate thousands of atoms to improve short-term stability, but single-atom detection and control remains an outstanding challenge. An ideal clock system, in this context, would thus merge the benefits of ion and lattice clocks, namely, a large array of isolated atoms that can be read out and controlled individually.

Here, we present a prototype of a new optical clock platform based on an atomic array, which naturally incorporates single-atom readout of currently about 40 individually trapped neutral atoms. Specifically, we use a magicwavelength 81-site tweezer array stochastically filled with single strontium-88 $\left({ }^{88} \mathrm{Sr}\right)$ atoms [25]. Employing a repetitive imaging scheme [25], we stabilize a local oscillator to the optical clock transition [26,27] with a low dead time of approximately $100 \mathrm{~ms}$ between clock interrogation blocks.

We utilize single-site and single-atom resolution to evaluate the in-loop performance of our clock system in terms of stability, local frequency shifts, selected systematic 
effects, and statistical properties. To this end, we define an error signal for single tweezers, which we use to measure site-resolved frequency shifts at otherwise fixed parameters. We also evaluate statistical properties of the in-loop error signal, specifically, the dependence of its variance on atom number and correlations between even and odd sites.

We further implement a standard interleaved selfcomparison technique [28,29] to evaluate systematic frequency shifts with changing external parametersspecifically, trap depth and wavelength - and find an operational magic condition [30-32] where the dependence on trap depth is minimized. We also demonstrate a proof of principle for extending such self-comparison techniques to evaluate single-site-resolved systematic frequency shifts as a function of a changing external parameter.

Using self-comparison, we evaluate the fractional shortterm instability of our clock system to be $2.5 \times 10^{-15} / \sqrt{\tau}$. To compare our experimental results with theory predictions, we develop an $a b$ initio Monte Carlo (MC) clock simulation [33] (Appendix A), which directly incorporates laser noise, projective readout, finite temperature, and feedback dynamics, resulting in higher predictive power compared to traditionally used analytical methods [1]. Our experimental data agree quantitatively with this simulation, indicating that noise processes are well captured and understood at the level of stability we achieve here. Based on the MC model, we predict a fractional instability of $(1.9-2.2) \times 10^{-15} / \sqrt{\tau}$ for single-clock operation, which would have shorter dead time than that in self-comparison.

We further demonstrate a direct evaluation of the $1 / \sqrt{N_{A}}$ dependence of clock stability with atom number $N_{A}$, on top of a laser-noise-dominated background, through an atom-byatom system-size-selection technique. This measurement and the MC model strongly indicate that the instability is limited by the frequency noise of our local oscillator. We note that the measured instability is comparable to OLCs using similar transportable laser systems [34].

We note the very recent, complementary results of Ref. [35] that show seconds-long coherence in a tweezer array filled with approximately $5{ }^{88} \mathrm{Sr}$ atoms using an ultralow-noise laser without feedback operation. In this and our system, a recently developed repetitive interrogation protocol [25], similar to that used in ion clocks, provides a short dead time of approximately $100 \mathrm{~ms}$ between interrogation blocks, generally suppressing the impact of laser noise on stability stemming from the Dick effect [36]. Utilizing seconds-scale interrogation with such low dead times, combined with the feedback operation and realistic upgrade to the system size demonstrated here, promises a clock stability that could reach that of state-of-the-art OLCs $[2,4,37,38]$ in the near-term future, as further discussed in Sec. VI.

Concerning systematic effects, the demonstrated atomic array clock has intrinsically suppressed interaction and hopping shifts: First, single-atom trapping in tweezers provides immunity to on-site collisions present in onedimensional OLCs [39]. While three-dimensional OLCs [37] also suppress on-site collisions, our approach retains a short dead time as no evaporative cooling is needed. Furthermore, the adjustable and significantly larger interatomic spacing strongly reduces dipolar interactions [40] and hopping effects [41]. We experimentally study effects from tweezer trapping in Sec. IV and develop a corresponding theoretical model in Appendix E, but we leave a full study of other systematics, not specific to our platform, and a statement of accuracy to future work. In this context, we note that our tweezer system is well suited for future investigations of blackbody radiation shifts via the use of local thermometry with Rydberg states [14].

The results presented here and in Ref. [35] provide the foundation for establishing a third optical clock platform promising competitive stability, accuracy, and robustness, while incorporating single-atom detection and control techniques in a natural fashion. We expect this to be a crucial development for applications requiring advanced control and readout techniques in many-atom quantum systems, as discussed in more detail in Sec. VI.

\section{FUNCTIONAL PRINCIPLE}

The basic functional principle is as follows. We generate a tweezer array with linear polarization and $2.5-\mu \mathrm{m}$ site-to-site spacing in an ultrahigh-vacuum glass cell using an AOD and a high-resolution imaging system [Fig. 1(a)] [25]. The tweezer-array wavelength is tuned to a magic trapping configuration close to $813.4 \mathrm{~nm}$, as described below. We load the array from a cold atomic cloud and subsequently induce light-assisted collisions to eliminate higher trap occupancies [25,42]. As a result, approximately 40 of the tweezers are stochastically filled with a single atom. We use a recently demonstrated narrow-line Sisyphus cooling scheme [25] to cool the atoms to an average transverse motional occupation number of $\bar{n} \approx 0.66$, measured with clock sideband spectroscopy (Appendix B 7). The atoms are then interrogated twice on the clock transition, once below $(A)$ and once above $(B)$ resonance, to obtain an error signal quantifying the frequency offset from the resonance center [Figs. 1(b) and 1(c)]. We use this error signal to feedback to a frequency shifter in order to stabilize the frequency of the interrogation laser-acting as a local oscillator-to the atomic clock transition. Since our imaging scheme has a survival fraction of greater than 0.998 [25], we perform multiple feedback cycles before reloading the array, each composed of a series of cooling, interrogation, and readout blocks [Fig. 1(d)].

For state-resolved readout with single-shot, single-atom resolution, we use a detection scheme composed of two high-resolution images for each of the $A$ and $B$ interrogation blocks [Fig. 1(e)] [25]. A first image determines if a tweezer is occupied, followed by clock interrogation. 


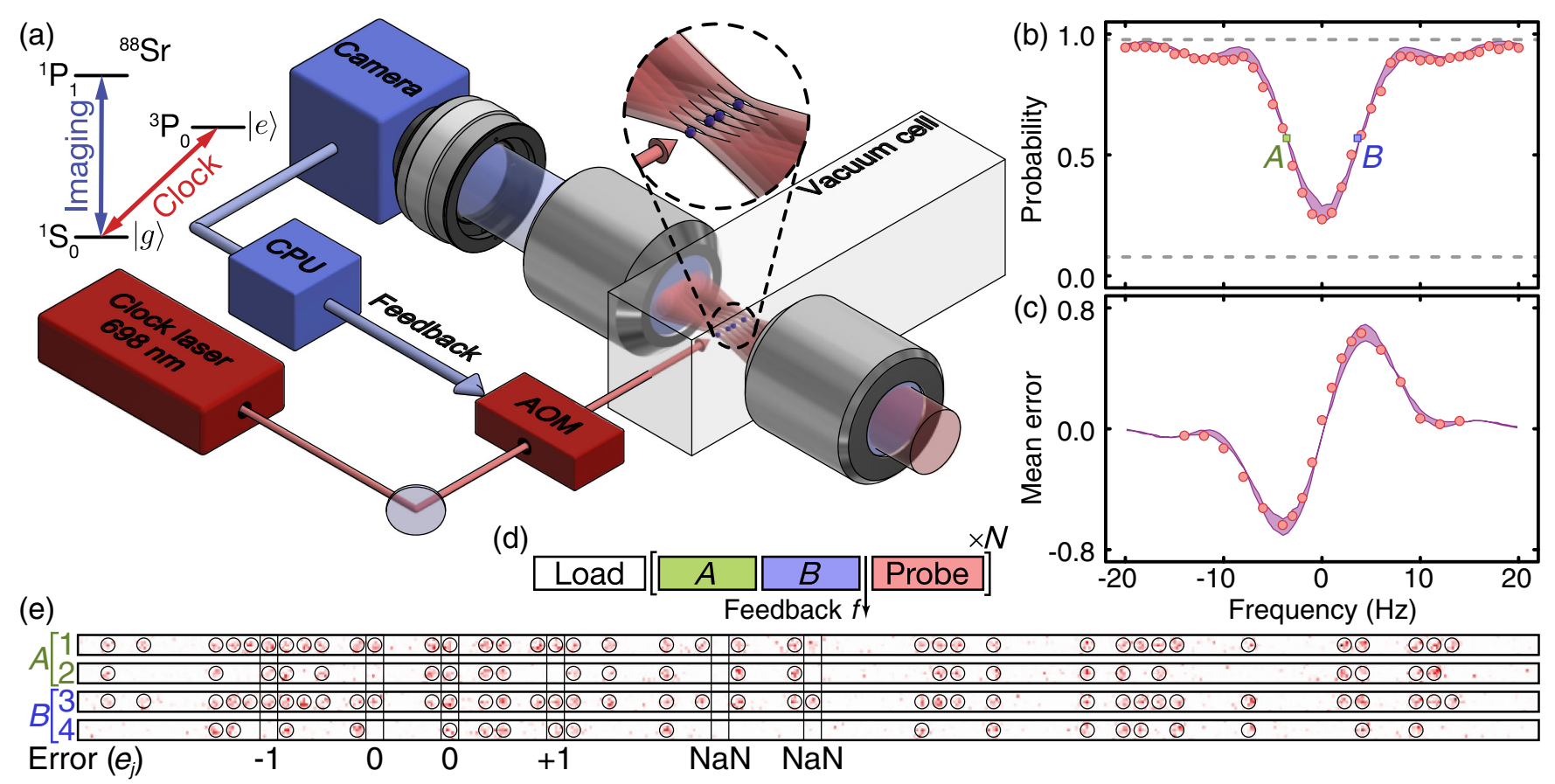

FIG. 1. Atomic array optical clock. (a) We interrogate about $40{ }^{88} \mathrm{Sr}$ atoms, trapped in an 81 -site tweezer array, on the ultranarrow clock transition at $698 \mathrm{~nm}$ and use high-resolution fluorescence imaging at $461 \mathrm{~nm}$ to detect population changes in the clock states (labeled $|g\rangle$ and $|e\rangle$ ) with single-atom resolution. This information is processed by a central processing unit (CPU), and a feedback signal is applied to the clock laser frequency using an acousto-optic modulator (AOM). (b) Tweezer-averaged probability to remain in $|g\rangle$ as a function of frequency offset measured with an in-loop probe sequence (circles). Dashed horizontal lines indicate state-resolved detection fidelities (Appendix B 5). To generate an error signal, we interrogate twice: once below (A) and once above (B) resonance. (c) Tweezeraveraged error signal as a function of frequency offset (circles). The shaded areas in (b) and (c) show results from MC simulations. (d) Simplified experimental sequence, consisting of tweezer loading and $N$-times-repeated $A B$ feedback blocks followed by an optional probe block, with $N=10$ throughout. (e) To detect the clock state population in block $A$, we take a first image before interrogation to identify which tweezers are occupied and a second image after interrogation to detect which atoms remain in $|g\rangle$ (images 1 and 2 ). The same procedure is repeated for block $B$ (images 3 and 4). We show fluorescence images with identified atoms (circles) (Appendix B 4) and examples of single tweezer error signals $e_{j}$.

A second image, after interrogation, determines if the atom has remained in the ground state $|g\rangle$. This process yields an instance of an error signal for all tweezers that are occupied at the beginning of both interrogation blocks, while unoccupied tweezers are discounted. For occupied tweezers, we record the $|g\rangle$ occupation numbers $s_{A, j}=\{0,1\}$ and $s_{B, j}=\{0,1\}$ in the images after interrogation with $A$ and $B$, respectively, where $j$ is the tweezer index. The difference $e_{j}=s_{A, j}-s_{B, j}$ defines a single-tweezer error variable taking on three possible values, $e_{j}=\{-1,0,+1\}$, indicating interrogation below, on, or above resonance, respectively. Note that the average of $e_{j}$ over many interrogations, $\left\langle e_{j}\right\rangle$, is simply an estimator for the difference in transition probability between blocks $A$ and $B$.

For feedback to the clock laser, $e_{j}$ is averaged over all occupied sites in a single $A B$ interrogation cycle, yielding an array-averaged error $\bar{e}=\left(1 / N_{A}\right) \sum_{j} e_{j}$, where the sum runs over all occupied tweezers and $N_{A}$ is the number of atoms present. We add $\bar{e}$ times a multiplicative factor to the frequency shifter, with the magnitude of this factor optimized to minimize in-loop noise.

\section{IN-LOOP SPECTROSCOPIC RESULTS}

We begin by describing results for in-loop detection sequences. Here, feedback is applied to the clock laser (as described before), and probe blocks, for which the interrogation frequency is varied, are added after each feedback cycle. Using a single probe block with an interrogation time of $110 \mathrm{~ms}$ (corresponding to a $\pi$ pulse on resonance) shows a nearly Fourier-limited line shape with full width at half maximum of approximately $7 \mathrm{~Hz}$ [Fig. 1(b)]. We also use these parameters for the feedback interrogation blocks, with the $A$ and $B$ interrogation frequencies spaced by a total of $7.6 \mathrm{~Hz}$. Using the same in-loop detection sequence, we can also directly reveal the shape of the error signal by using two subsequent probe blocks spaced by this frequency difference and scanning a common frequency offset [Fig. 1(c)]. The experimental results are in agreement with MC simulations, which have systematic error denoted as a shaded area throughout, stemming from uncertainty in the noise properties of the interrogation laser (Appendix A 3).

Importantly, these data also exist on the level of individual tweezers, both in terms of averages and 
statistical fluctuations. As a first example, we show a tweezer-resolved measurement of the repetition-averaged error signal $\left\langle e_{j}\right\rangle$ for all 81 traps [Fig. 2(a)] as a function of frequency offset.

Fitting the zero crossings of $\left\langle e_{j}\right\rangle$ enables us to detect differences in resonance frequency with sub- $\mathrm{Hz}$ resolution [Fig. 2(b)]. The results show a small gradient across the array due to the use of an AOD: Tweezers are spaced by $500 \mathrm{kHz}$ in optical frequency, resulting in an approximately linear variation of the clock transition frequency. This effect

\section{(a)}
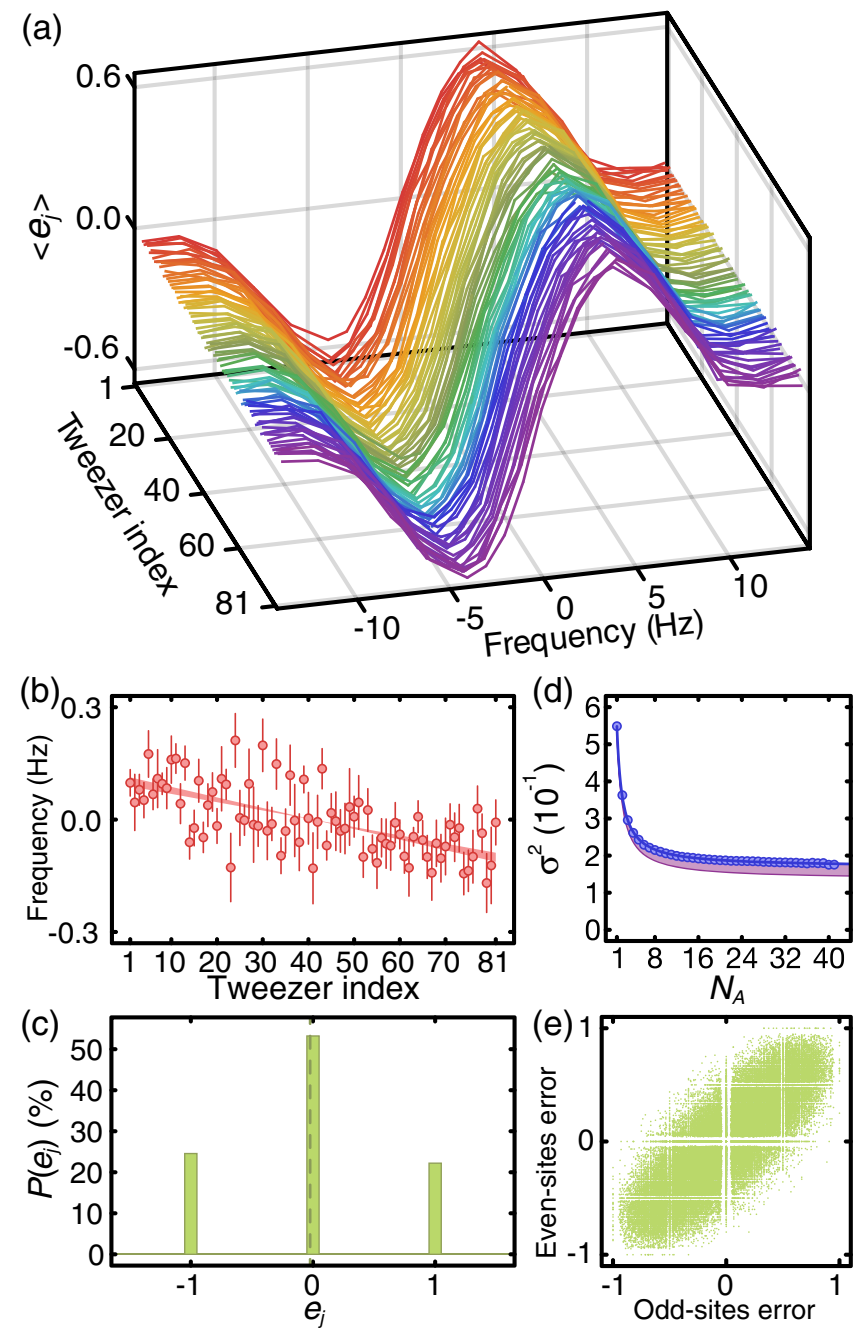

FIG. 2. Site-resolved error signal. (a) Repetition-averaged single-tweezer error signal $\left\langle e_{j}\right\rangle$ as a function of frequency offset measured with an in-loop sequence. (b) Fitted zero crossings as a function of tweezer index for our usual interrogation trap depth of $U_{1}=245(31) E_{r}$, where $E_{r}=h \times 3.43 \mathrm{kHz}$ (circles). Solid lines correspond to theory predictions, with the shaded area resulting from systematic uncertainty in trap depth (Appendix E). (c) Ternary probability distribution for $e_{j}$ for a selected tweezer. The vertical dashed line shows the mean. (d) Variance of the error signal as a function of atom number, calculated through postselection. The solid line is a fit with a $1 / N_{A}$ function plus an offset. The purple region is a MC simulation. (e) Plot of correlations between the error signals of even and odd sites. could be avoided by using a spatial light modulator for tweezer-array generation [43]. We note that the total frequency variation is smaller than the width of our interrogation signal. Such "subbandwidth" gradients can still lead to noise through stochastic occupation of sites with slightly different frequencies; in our case, we predict an effect at the $10^{-17}$ level. We propose a method to eliminate this type of noise in future clock iterations with a local feedback correction factor in Appendix D 3.

Before moving on, we note that $e_{j}$ is a random variable with a ternary probability distribution [Fig. 2(c)] defined for each tweezer. The results in Fig. 2(a) are the mean of this distribution as a function of frequency offset. In addition to such averages, having a fully site-resolved signal enables valuable statistical analysis. As an example, we extract the variance of $\bar{e}, \sigma_{\bar{e}}^{2}$, for an in-loop probe sequence where the probe blocks are centered around resonance.

Varying the number of atoms taken into account (via postselection) shows a $1 / N_{A}$ scaling with a prefactor dominated by quantum projection noise (QPN) [1] on top of an offset stemming mainly from laser noise [Fig. 2(d)]. A more detailed analysis reveals that, for our atom number, the relative noise contribution from QPN to $\sigma_{\bar{e}}$ is only approximately $26 \%$ (Appendix C). A similar conclusion can be drawn on a qualitative level by evaluating correlations between tweezer-resolved errors from odd and even sites, which show a strong common mode contribution indicative of sizable laser noise [Fig. 2(e)].

\section{SELF-COMPARISON FOR EVALUATION OF SYSTEMATIC SHIFTS FROM TWEEZER TRAPPING}

We now turn to an interleaved self-comparison [28,29], which we use for stability evaluation and systematic studies. The self-comparison consists of having two feedback loops running in parallel, where feedback is given in an alternating fashion to update two independent AOM frequencies, $f_{1}$ and $f_{2}$ [Fig. 3(a)]. This self-comparison is used for a lock-in-type evaluation of clock frequency changes with varying parameters. As a specific example, we operate the clock with our usual interrogation trap depth $U_{1}$ during blocks for feedback to $f_{1}$ and with a different trap depth $U_{2}$ during blocks for feedback to $f_{2}$. The average frequency difference $f_{2}-f_{1}$ now reveals a shift of the clock-operation frequency dependent on $U_{2}$ [Fig. 3(b)]. For optimal clock operation, we find an "operationally magic" condition that minimizes sensitivity to trap-depth fluctuations [30-32] by performing two-lock comparisons for different wavelengths [Fig. 3(b)] (Appendix E). We note that this type of standard self-comparison can only reveal array-averaged shifts.

In this context, an important question is how such lock-in techniques can be extended to reveal site-resolved systematic errors as a function of a changing external parameter. 

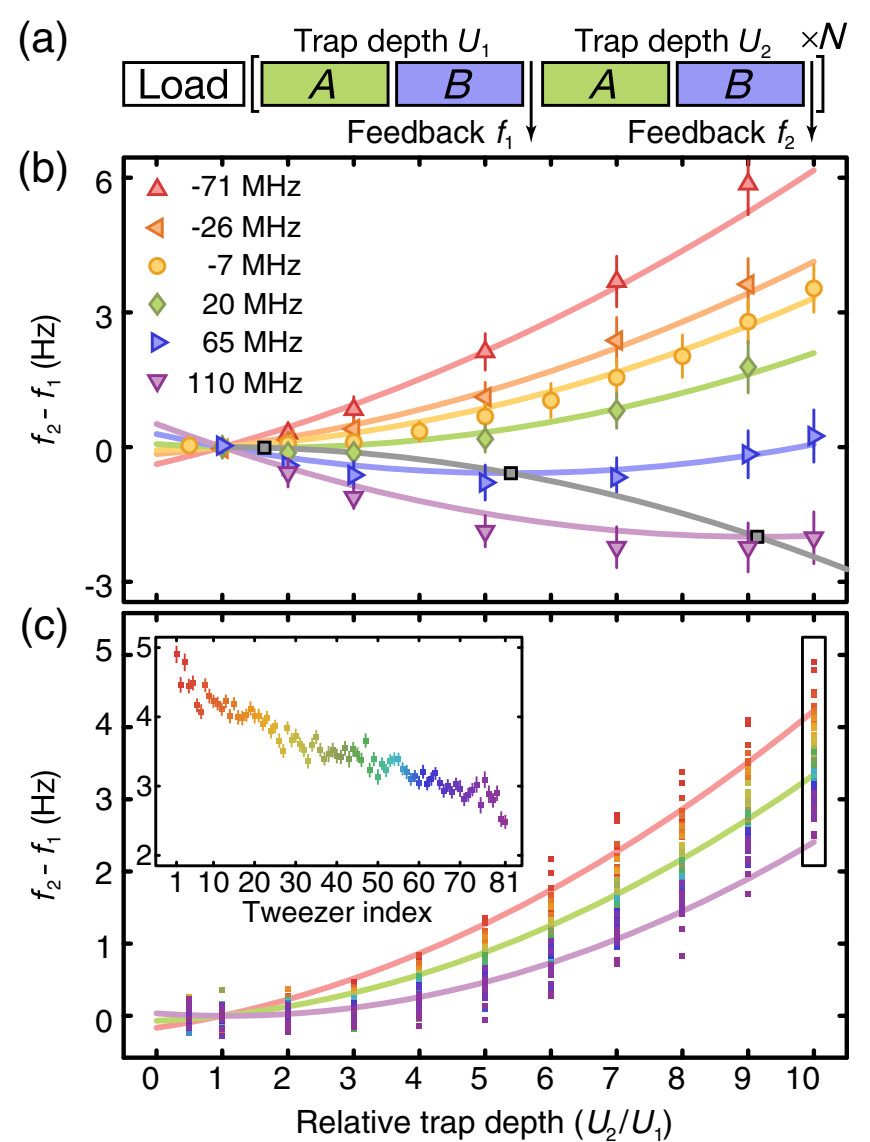

FIG. 3. Systematic evaluation of clock shifts with tweezer depth and wavelength. (a) Illustration of interleaved self-comparison, where two independent AOM frequencies $\left(f_{1}\right.$ and $\left.f_{2}\right)$ are updated in an alternating fashion. Respective interrogation blocks are set to two independent tweezer depths $U_{1}$ and $U_{2}$. (b) Average frequency difference $f_{2}-f_{1}$ as a function of $U_{2} / U_{1}$, with $U_{1}$ fixed to our usual interrogation depth, for multiple frequency offsets of the trapping laser (see legend for color coding). We fit the data with a model for light shifts in optical tweezers (colored lines) with only a single free parameter (for all data simultaneously), accounting for an unknown frequency offset (Appendix E). Operational magic intensities are found at the minima of these curves (gray squares and connecting line), which minimize the sensitivity to trap-depth fluctuations. The trap laser frequency is tuned such that the minimum coincides with our nominal depth. (c) Combining this technique with the single-tweezerresolved error $\left\langle e_{j}\right\rangle$, we can extract a frequency dependence with trap depth for each tweezer (colored squares). Solid lines show the expected dependence for the outermost and central tweezers. The data correspond to the $-7 \mathrm{MHz}$ set in (b). Inset: Local frequency shifts for $U_{2} / U_{1}=10$. The color coding of the inset defines the color coding of its subfigure.

To this end, we combine the tweezer-resolved error signal $\left\langle e_{j}\right\rangle$ with interleaved self-comparison [Fig. 3(c)]. Converting $\left\langle e_{j}\right\rangle$ to frequencies [using measured error functions, such as in Fig. 2(a)] yields frequency estimators $\delta f_{1, j}$ and $\delta f_{2, j}$ for each tweezer during $f_{1}$ and $f_{2}$ feedback blocks, respectively. These estimators correspond to the relative resonance frequency of each tweezer with respect to the center frequency of the individual locks. Plotting the quantity $\delta f_{2, j}-\delta f_{1, j}+f_{2}-f_{1}$ then shows the absolute frequency change of each tweezer as a function of trap depth [Fig. 3(c)].

\section{SELF-COMPARISON FOR STABILITY EVALUATION}

We use the same self-comparison sequence to evaluate the fractional clock instability by operating both locks with identical conditions [Fig. 4(a)]. This approach follows previous clock studies, where true comparison to a second, fully independent clock system was not available $[28,29]$. We plot the Allan deviation $\sigma_{y}$ [44] of
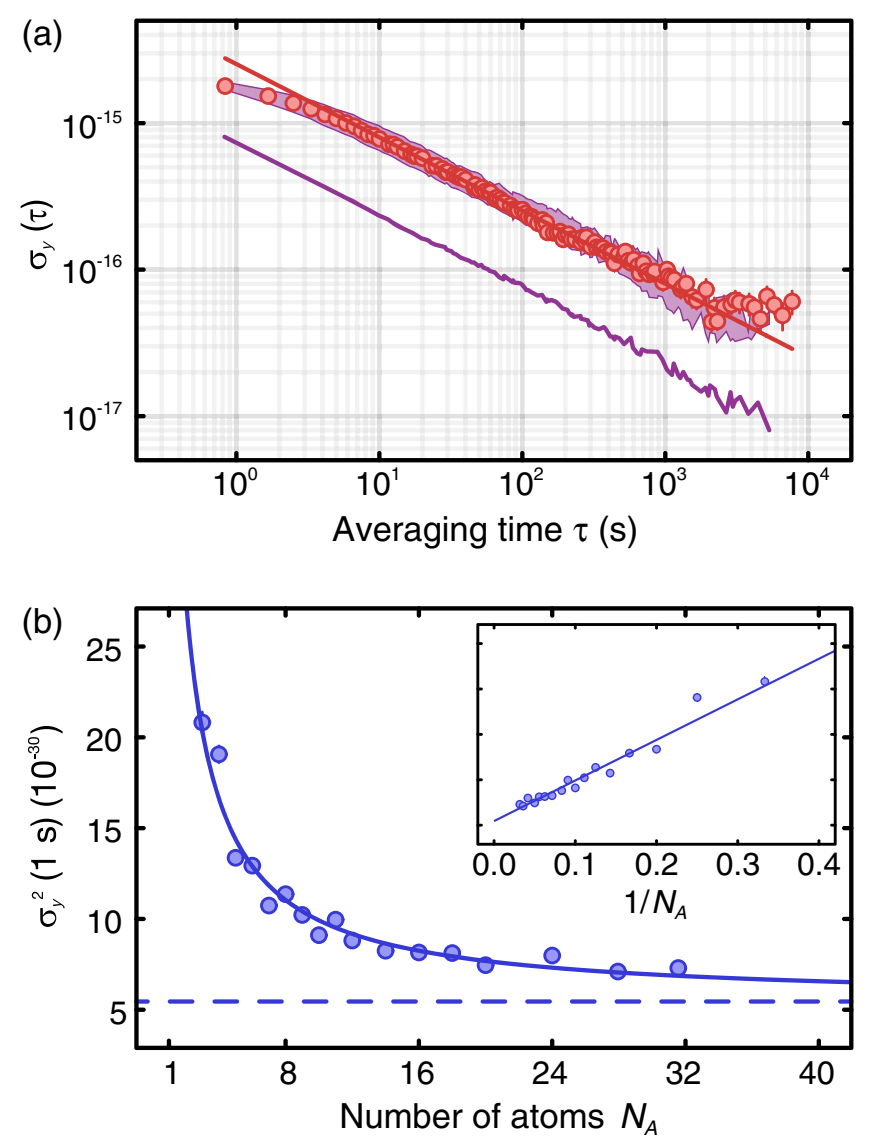

FIG. 4. Stability results. (a) Fractional Allan deviation $\sigma_{y}$ obtained via self-comparison as a function of integration time $\tau$ (circles). Fitting a $1 / \sqrt{\tau}$ behavior past an initial lock onset time (red solid line), we find $2.5 \times 10^{-15} / \sqrt{\tau}$. The shaded area denotes MC results. The purple solid line shows the quantum projection noise limit obtained from MC by switching off all other noise sources. (b) Based on atom-by-atom feedback control, we perform a series of self-comparisons with fixed atom number $N_{A}$. Shown is the Allan variance $\sigma_{y}^{2}$ at 1 second (from a $1 / \sqrt{\tau}$ fit) as a function of $N_{A}$. Inset: Allan variance $\sigma_{y}^{2}$ as a function of $1 / N_{A}$. Solid lines show a fit with a functional form $\sigma_{y}^{2}=\sigma_{\infty}^{2}+\sigma_{N_{A}}^{2}$, where $\sigma_{N_{A}}$ scales as $1 / \sqrt{N_{A}}$. 
$y=\left(f_{2}-f_{1}\right) /\left(\nu_{0} \sqrt{2}\right)$ in Fig. 4(a), where $\nu_{0}$ is the clock transition frequency and the $\sqrt{2}$ factor is introduced to take into account the addition of noise from two identical sources. The results show a $1 / \sqrt{\tau}$ behavior after a lock onset time, where $\tau$ is the averaging time in seconds. Fitting this behavior yields $\sigma_{y}=2.5 \times 10^{-15} / \sqrt{\tau}$, in excellent agreement with MC simulations [Fig. 4(a)].

Self-comparison evaluates how fast averaging can be performed for systematic studies-such as the one shown in Fig. 3-and reveals the impact of various noise sources on short-term stability; however, by design, this technique suppresses slow drifts that are common to the $f_{1}$ and $f_{2}$ interrogation blocks. We performed a separate stability analysis by locking $f_{1}$ to the left half of the array and $f_{2}$ to the right half of the array [37], a method that is sensitive to slow drifts of gradients, and found no long-term drift of gradients to within our sensitivity (Appendix D 2).

Having shown good agreement between our data and MC simulations, we are able to further use the simulation to predict properties of our clock that are not directly experimentally accessible. One of these properties is the true stability of the local oscillator frequency, computed directly by taking the Allan deviation of the simulated laser-frequency time traces under feedback. This method allows us to simulate the stability of single-clock operation, which has shorter dead time than the double clock scheme that we use to evaluate stability in experiment. Following this protocol, our simulations predict $(1.9-2.2) \times 10^{-15} / \sqrt{\tau}$ for the local oscillator stability during single-clock operation (Appendix A). In this context, we note the results of Ref. [35], where stability is evaluated by converting a spectroscopic signal into a frequency record (without a closed feedback loop). Based on interrogation with an ultralow-noise laser system, they achieve a short-term stability of $4.7 \times 10^{-16} / \sqrt{\tau}$, with approximately 5 atoms in tweezers.

Generically, clock stability improves with increasing atom number as $1 / \sqrt{N_{A}}$ through a reduction in readout noise as long as atoms are uncorrelated. However, in the presence of laser noise-which is common mode to all atoms - a limit to stability exists even for an infinite number of atoms [1]. Intriguingly, we can directly extract such contributions by performing a series of selfcomparisons, where we adjust the atom number one by one [Fig. 4(b)]. To this end, we restrict the feedback operation to a subset of atoms in the center of the array with desired size, ignoring the remainder. We are able to achieve stable locking conditions for $N_{A} \geq 3$ with typical feedback parameters. We evaluate the Allan variance at 1 second as a function of $N_{A}$ and fit the results with a function $\sigma_{y}^{2}=\sigma_{\infty}^{2}+\sigma_{N_{A}}^{2}$, where $\sigma_{N_{A}}$ scales as $1 / \sqrt{N_{A}}$. We find $\sigma_{N_{A}}=6.7 \times 10^{-15} / \sqrt{N_{A} \cdot \tau}$ and $\sigma_{\infty}=2.3 \times 10^{-15} / \sqrt{\tau}$, the latter being an estimator for the limit of our clock set by laser noise, in agreement with MC simulation.

\section{OUTLOOK}

Our results merge single-particle readout and control techniques for neutral atom arrays with optical clocks based on ultranarrow spectroscopy. Such atomic array optical clocks (AOCs) could approach the sub-10 $-16 / \sqrt{\tau}$ level of stability achieved with OLCs $[2,4,37,38]$ by increasing interrogation time and atom number. Reaching several hundreds of atoms is realistic with an upgrade to twodimensional arrays, while Ref. [35] already demonstrated seconds-long interrogation. A further increase in atom number is possible by using a secondary array for readout, created with a nonmagic wavelength for which higherpower lasers exist $[42,45]$. We also envision a system where tweezers are used to "implant" atoms, in a structured fashion, into an optical lattice for interrogation and are subsequently used to provide confinement for single-atom readout. Furthermore, the lower dead time of AOCs should help us to reduce laser noise contributions to clock stability compared to 3D OLCs [37], and even zero dead time operation $[37,38]$ in a single machine is conceivable by adding local interrogation. Local interrogation could be achieved by addressing through the main objective or through an orthogonal high-resolution path using spatial-light modulators or acousto-optic devices. For the case of addressing through the main objective, atoms would likely need to be trapped in an additional lattice to increase longitudinal trapping frequencies.

Concerning systematics, AOCs provide fully siteresolved evaluation combined with an essential mitigation of interaction shifts while being ready-made for implementing local thermometry using Rydberg states [14] in order to more precisely determine blackbody-induced shifts [1]. In addition, AOCs offer an advanced toolset for generation and detection of entanglement to reach beyond standard quantum limit operation-either through cavities $[16,46]$ or Rydberg excitation [15] — and for implementing quantum clock networks [19]. Furthermore, the demonstrated techniques provide a pathway for quantum computing and communication with neutral alkaline-earth-like atoms [8,20,22]. Finally, features of atomic array clocks - such as experimental simplicity, short dead time, and three-dimensional confinement-make these systems attractive candidates for robust portable clock systems and space-based missions [31].

\section{ACKNOWLEDGMENTS}

We acknowledge funding provided by the Institute for Quantum Information and Matter, a NSF Physics Frontiers Center (NSF Grant No. PHY-1733907). This work was supported by the NSF (Grant No. 1753386), the AFOSR YIP (Grant No. FA9550-19-1-0044), and the Sloan Foundation. This research was carried out at the Jet Propulsion Laboratory and the California Institute of Technology under a contract with the National Aeronautics 
and Space Administration and funded through the Presidents and Directors Research and Development Fund (PDRDF). T. H. Y. acknowledges support from the National Research Foundation of Korea Grant No. NRF2019009974. We acknowledge generous support from Fred Blum.

\section{APPENDIX A: MONTE CARLO SIMULATION}

\section{Operation}

We compare the performance of our clock to $\mathrm{MC}$ simulations. The simulations include the effects of laserfrequency noise, dead time during loading and between interrogations, quantum projection noise, finite temperature, stochastic filling of tweezers, and experimental imperfections such as state-detection infidelity and atom loss. The effects of Raman scattering from the trap and of differential trapping due to hyperpolarizability or trap wavelength shifts from the AOD are not included as they are not expected to be significant at our level of stability.

Rabi interrogation is simulated by time evolving an initial state $|g\rangle$ with the time-dependent Hamiltonian $\hat{H}(t)=(\hbar / 2)\left\{\Omega \sigma_{x}+\left[\delta(t) \pm \delta_{o}\right] \sigma_{z}\right\}$, where $\Omega$ is the Rabi frequency, $\delta_{o}$ is an interrogation offset, and $\delta(t)$ is the instantaneous frequency noise defined such that $\delta(t)=[d \phi(t)] / d t$, where $\phi(t)$ is the optical phase in the rotating frame. The frequency noise $\delta(t)$ for each Rabi interrogation is sampled from a pregenerated noise trace (Appendixes A 2 and A 3) with a discrete time step of $10 \mathrm{~ms}$. Dead time between interrogations and between array refilling is simulated by sampling from timeseparated intervals of this noise trace. Stochastic filling is implemented by sampling the number of atoms $N_{A}$ from a binomial distribution on each filling cycle, and atom loss is implemented by probabilistically reducing $N_{A}$ between interrogations.

To simulate finite temperature, a motional quantum number $n$ is assigned to each of the $N_{A}$ atoms before each interrogation, where $n$ is sampled from a 1D thermal distribution using our experimentally measured $\bar{n} \approx 0.66$ (Appendix B 7). Here, $n$ represents the motional quantum number along the axis of the interrogating clock beam. For each of the unique values of $n$ that were sampled, a separate Hamiltonian evolution is carried out with a modified Rabi frequency given by $\Omega_{n}=\Omega e^{-\left(\eta^{2} / 2\right)} L_{n}\left(\eta^{2}\right)$ [47], where $\eta=\left[(2 \pi) / \lambda_{\text {clock }}\right] \sqrt{[\hbar /(2 m \omega)]}$ is the LambDicke parameter, $L_{n}$ is the $n$th order Laguerre polynomial, and $\Omega$ is the bare Rabi frequency valid in the limit of infinitely tight confinement.

At the end of each interrogation, excitation probabilities $p_{e}(n)=\left|\left\langle e \mid \psi_{n}\right\rangle\right|^{2}$ are computed from the final states for each $n$. State-detection infidelity is simulated by defining adjusted excitation probabilities $\tilde{p}_{e}(n) \equiv f_{e} p_{e}(n)+$ $\left(1-f_{g}\right)\left(1-p_{e}(n)\right)$, where $f_{g}$ and $f_{e}$ are the groundand excited-state detection fidelities (Appendix B 5), respectively. To simulate readout of the $j$ th atom on the $i$ th interrogation, a Bernoulli trial with probability $\tilde{p}_{e}\left(n_{j}\right)$ is performed, producing a binary readout value $s_{j, i}$. An error signal $\bar{e}=\left(1 / N_{A}\right) \sum_{j}\left(s_{j, i-1}-s_{j, i}\right)$ is produced every two interrogation cycles by alternating the sign of $\delta_{o}$ on alternating interrogation cycles. This error signal produces a control signal (using the same gain factor as used in experiment), which is summed with the generated noise trace for the next interrogation cycle, closing the feedback loop.

\section{Generating frequency noise traces}

Using a model of the power spectral density of our clock laser's frequency noise (Appendix A 3), we generate random frequency noise traces in the time domain [48] for use in the Monte Carlo simulation. Given the power spectral density of frequency noise $S_{\nu}(f)$, we generate a complex one-sided amplitude spectrum $A_{\nu}(f)=$ $e^{i \phi(f)} \sqrt{2 S_{\nu}(f) \Delta f}$, where $\phi(f)$ is sampled from a uniform distribution in $[0,2 \pi)$ for each $f$ and $\Delta f$ is the frequency discretization. This spectrum is converted to a two-sided amplitude spectrum by defining $A_{\nu}(-f)=A_{\nu}^{*}(f)$. Finally, a time trace $\nu(t)=\mathcal{F}\{A(f)\}(t)+\nu_{l}(t)$ is produced by taking a fast Fourier transform (FFT) of $A(f)$ and adding an experimentally calibrated linear drift term $\nu_{l}(t)$.

\section{Frequency noise model}

The power spectral density of the frequency noise of our clock laser is modeled by the sum of contributions from random walk frequency modulation (RWFM) noise $\left(f^{-2}\right)$, flicker frequency modulation (FFM) noise $\left(f^{-1}\right)$, and white frequency modulation (WFM) noise $\left(f^{0}\right)$, such that $S_{\nu}(f)=\alpha f^{-2}+\beta f^{-1}+\gamma f^{0}$. We obtain these parameters through an estimation of the thermal noise of our reference cavity and a fit of a partially specified frequency-noise power spectral density obtained via beating our laser with a reference laser (Fig. 5). Because of the remaining large uncertainty in the white noise floor of our laser, we define worst-case and best-case noise models. The range between these models is the dominant source of uncertainty in our Monte Carlo simulations.

FFM noise results from thermal mechanical fluctuations of the reference cavity [49,50]. By estimating the noise contribution from the ultralow-expansion spacer, fused silica mirrors, and their reflective coating, we estimate a fractional frequency instability of $\sigma_{y}=1.6 \times 10^{-15}$ at $1 \mathrm{~s}$, which corresponds to a frequency-noise power spectral density of $\beta f^{-1}=0.34 \mathrm{~Hz}^{2} / \mathrm{Hz}$ at $f=1 \mathrm{~Hz}$.

As a worst-case noise model, we assume a crossover frequency from FFM to WFM noise at $1 \mathrm{~Hz}$ (Fig. 5), such that $\gamma=\beta f^{-1}=0.34 \mathrm{~Hz}^{2} / \mathrm{Hz}$, and we estimate a frequency-noise power spectral density of $\alpha f^{-2}=$ $0.05 \mathrm{~Hz}^{2} / \mathrm{Hz}$ at $1 \mathrm{~Hz}$ for RWFM noise. As a best-case 


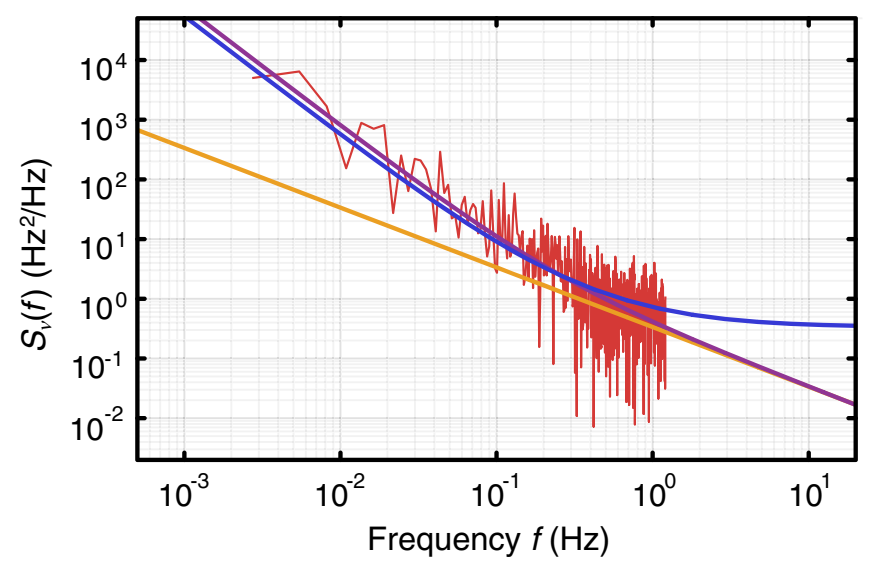

FIG. 5. Frequency noise spectrum of the clock laser. Power spectral density of the frequency noise of our clock laser measured from a beat signal with a reference laser over a 42 hour period (red trace). Our theoretical estimate of the thermal noise contribution is plotted in yellow. We also plot our best-case (purple) and worst-case (blue) models for total frequency noise, as used in Monte Carlo simulations.

noise model, assuming no crossover from FFM to WFM noise (such that $\gamma=0.00 \mathrm{~Hz}^{2} / \mathrm{Hz}$ ), we estimate a frequency-noise power spectral density for RWFM noise of $\alpha f^{-2}=0.08 \mathrm{~Hz}^{2} / \mathrm{Hz}$ at $f=1 \mathrm{~Hz}$. We note that the difference in predicted clock stability between the best- and worst-case models is relatively minor. This minor difference indicates that dominant contributions to clock instability stem from frequencies where we have experimental frequency noise data and where both models exhibit similar frequency noise. This case is confirmed by an analytical Dick noise analysis [36] (not shown).

\section{APPENDIX B: EXPERIMENTAL DETAILS}

\section{Experimental system}

Our strontium apparatus is described in detail in Refs. [25,42]. Strontium-88 atoms from an atomic beam oven are slowed and cooled to a few microkelvin temperature by a $3 \mathrm{D}$ magneto-optical trap operating first on the broad dipole-allowed ${ }^{1} \mathrm{~S}_{0} \leftrightarrow{ }^{1} \mathrm{P}_{1}$ transition at $461 \mathrm{~nm}$ and then on the narrow spin-forbidden ${ }^{1} \mathrm{~S}_{0} \leftrightarrow{ }^{3} \mathrm{P}_{1}$ transition at $689 \mathrm{~nm}$. Strontium atoms are filled into a 1D array of 81 optical tweezers at $\lambda_{T}=813.4 \mathrm{~nm}$, which is the magic wavelength for the doubly forbidden ${ }^{1} \mathrm{~S}_{0} \leftrightarrow{ }^{3} \mathrm{P}_{0}$ optical clock transition. The tweezers have Gaussian waist radii of $800(50) \mathrm{nm}$ and an array spacing of $2.5 \mu \mathrm{m}$. During filling, cooling, and imaging (state detection), the trap depth is $2447(306) E_{r}$. Here, $E_{r}$ is the tweezer photon recoil energy, given by $E_{r}=h^{2} /\left(2 m \lambda_{T}^{2}\right)$, where $h$ is Planck's constant and $m$ is the mass of ${ }^{88} \mathrm{Sr}$. The tweezer depth is determined from the measured waist and the radial trapping frequency found from sideband measurements on the clock transition (discussed in more detail in Appendix B 7). After parity projection, each tweezer has a 0.5 probability of containing a single atom, or otherwise being empty. Thus, the total number of atoms $N_{A}$ after each filling cycle of the experiment follows a binomial distribution with mean number of atoms $\bar{N}_{A}=40.5$.

\section{Clock laser system}

Our clock laser is based on a modified portable clock laser system (Stable Laser Systems) composed of an external cavity diode laser (Moglabs) stabilized to an isolated, high-finesse optical cavity using the PoundDrever-Hall scheme and electronic feedback to the laser diode current and piezoelectric transducer. The optical cavity is a 50-mm cubic cavity [51] made of ultralowexpansion glass maintained at the zero-crossing temperature of $40.53{ }^{\circ} \mathrm{C}$ with mirror substrates made of fused silica with a finesse of $F>300000$ at $698 \mathrm{~nm}$. The clock laser light passes through a first AOM in a double-pass configuration, injects an antireflection-coated laser diode (Sacher Lasertechnik GmbH, SAL-0705-020), passes through a second AOM, and goes through a 10-m-long fiber to the main experiment with a maximum output optical power of $20 \mathrm{~mW}$. The first AOM is used for shifting and stabilizing the frequency of the clock laser, whereas the second $\mathrm{AOM}$ is used for intensity-noise and fiber-noise cancellation. The clock laser light has a Gaussian waist radius of $600 \mu \mathrm{m}$ along the tweezer array. This large width is chosen to minimize gradients in clock intensity across the array arising from slight beam-angle misalignments.

\section{Bosonic clock transition}

Optical excitation of the ${ }^{1} \mathrm{~S}_{0} \leftrightarrow{ }^{3} \mathrm{P}_{0}$ clock transmission in a bosonic alkaline-earth-like atom is facilitated by applying a bias magnetic field $B$ [26]. This field creates a small admixture of ${ }^{3} \mathrm{P}_{1}$ into ${ }^{3} \mathrm{P}_{0}$ and results in a Rabi frequency of $\Omega_{R} / 2 \pi=\alpha \sqrt{I}|B|$, where $I$ is the intensity of the clock probe beam and $\alpha$ is the coupling constant. For ${ }^{88} \mathrm{Sr}, \alpha=$ $198 \mathrm{~Hz} / \mathrm{T}\left(\mathrm{mW} / \mathrm{cm}^{2}\right)^{1 / 2}$ [26]. The probe beam induces an ac Stark shift $\Delta \nu_{P}=k I$, where $k=-18 \mathrm{mHz} /\left(\mathrm{mW} / \mathrm{cm}^{2}\right)$ for ${ }^{88} \mathrm{Sr}$ [26]. The magnetic field gives rise to a quadratic Zeeman shift $\Delta \nu_{B}=\beta B^{2}$, where $\beta=-23.3 \mathrm{MHz} / \mathrm{T}^{2}$ for ${ }^{88} \mathrm{Sr}[26]$.

We choose $B \approx 900 \mu \mathrm{T}$, for which $\Delta \nu_{B} \approx-18.9 \mathrm{~Hz}$, and we choose $I \approx 1560 \mathrm{~mW} / \mathrm{cm}^{2}$, for which $\Delta \nu_{P} \approx-28.1 \mathrm{~Hz}$. The quoted values for $B$ and $I$ are experimentally calibrated by measuring $\Delta \nu_{B}$ and $\Delta \nu_{P}$ via two-clock self-comparison (Sec. IV), where the value of the systematic parameter in the second rail is varied. We fit the measured frequency shift to a quadratic model for the magnetic shift and to a linear model for the probe shift (not shown) and extrapolate both fits to the known zero values of the systematic parameters, thus extracting $\Delta \nu_{B}$ and $\Delta \nu_{P}$.

We note that our measured $\pi$ time of 110 ms (Fig. 6) is longer than what would be expected from the calibrated 


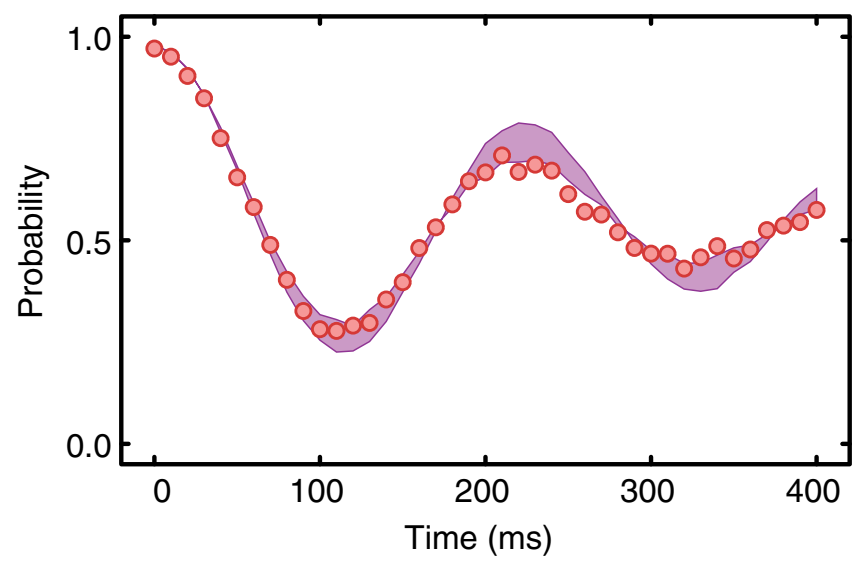

FIG. 6. Rabi oscillations on the clock transition with $\pi$-pulse length of $110 \mathrm{~ms}$. Each point is probed directly after stabilizing the clock laser with a feedback sequence as described in the main text. The shaded area denotes Monte Carlo results.

beam intensity. This result is likely explained by the spectral impurity of the interrogating light, which has servo-induced sidebands at approximately $600 \mathrm{kHz}$. These sidebands are spectrally resolved enough so as not to affect clock interrogation, but they still contribute to the probe light shift of the transition frequency.

\section{Interrogation sequence}

We confirm the presence of atoms in each tweezer using fluorescence imaging for $30 \mathrm{~ms}$ on the 461-nm transition while cooling on the $689-\mathrm{nm}$ transition and repumping atoms out of the metastable ${ }^{3} \mathrm{P}_{0,2}$ states. This imaging procedure initializes the atoms in the ${ }^{1} \mathrm{~S}_{0}$ electronic ground state $|g\rangle$. We then further cool the atoms for $10 \mathrm{~ms}$ using attractive Sisyphus cooling [25] on the 689-nm transition and adiabatically ramp down to a trap depth of 245(31) $E_{r}$ for $4 \mathrm{~ms}$. We apply a weak bias magnetic field of $B \approx$ $900 \mu \mathrm{T}$ along the transverse direction of the tweezer array to enable direct optical excitation of the doubly forbidden clock transition at $698 \mathrm{~nm}[26,52]$. After interrogating the clock transition for $110 \mathrm{~ms}$ (Fig. 6), we adiabatically ramp the trap depth back up to $2447(306) E_{r}$ to detect the population of atoms in $|g\rangle$ using fluorescence imaging for 30 ms without repumping on the ${ }^{3} \mathrm{P}_{0} \leftrightarrow{ }^{3} \mathrm{~S}_{1}$ transition. This interrogation sequence is repeated a number of times before the array is refilled with atoms.

\section{Clock state detection fidelity}

Based on the approach demonstrated in Ref. [25], we analyze the fidelity of detecting atoms in the ${ }^{1} \mathrm{~S}_{0}(|g\rangle)$ and ${ }^{3} \mathrm{P}_{0}(|e\rangle)$ states under these imaging conditions. We diagnose our state-detection fidelity with two consecutive images. In the first image, we detect atoms in $|g\rangle$ by turning off the ${ }^{3} \mathrm{P}_{0} \leftrightarrow{ }^{3} \mathrm{~S}_{1}$ repump laser such that atoms in $|e\rangle$, in principle, remain in $|e\rangle$ and do not scatter photons [25]. Hence, if we find a signal in the first image, we identify the state as $|g\rangle$. In the second image, we turn the ${ }^{3} \mathrm{P}_{0} \leftrightarrow{ }^{3} \mathrm{~S}_{1}$ repump laser back on to detect atoms in both $|g\rangle$ and $|e\rangle$. Thus, if an atom is not detected in the first image but appears in the second image, we can identify it as $|e\rangle$. If neither of the images shows a signal, we identify the state as "no atom".

The inaccuracy of this scheme is dominated by offresonant scattering of the tweezer light when atoms are shelved in $|e\rangle$ during the first image. By pumping atoms into $|e\rangle$ before imaging, we observe that they decay back to $|g\rangle$ with a time constant of $\tau_{p}=370(4) \mathrm{ms}$ at our imaging trap depth of $2447(306) E_{r}$. This result leads to events in the first image where $|e\rangle$ atoms are misidentified as $|g\rangle$ atoms. Additionally, atoms in $|g\rangle$ can be misidentified as $|e\rangle$ if they are pumped to $|e\rangle$ in the first image. We measure this misidentification probability by initializing atoms in $|g\rangle$ and counting how often we identify them as $|e\rangle$. Using this method, we place a lower bound for the probability of correctly identifying $|e\rangle$ as $f_{e} \equiv e^{-t / \tau_{p}}=0.922(1)$, and we directly measure the probability of correctly identifying $|g\rangle$ as $f_{g}=0.977(2)$. These values are shown in Fig. 1(b) as dashed lines.

\section{Stabilization to the atomic signal}

The clock laser is actively stabilized to the atomic signal using a digital control system. The frequency deviation of the clock laser from the atomic transition is estimated from a two-point measurement of the Rabi spectroscopy signal at $\delta_{o} / 2 \pi= \pm 3.8 \mathrm{~Hz}$ for an interrogation time of $110 \mathrm{~ms}$, which produces an experimentally measured line shape with a full width at half maximum of $7 \mathrm{~Hz}$. Here, $\bar{e}$ is converted into a frequency correction by multiplying it by a factor of $\kappa=3 \mathrm{~Hz}$. We choose $\kappa$ to be the largest value possible before the variance of the error signal in an in-loop probe sequence begins to grow. Feedback is performed by adding the frequency correction to the frequency of the $\mathrm{rf}$ synthesizer (Moglabs ARF421) driving the first AOM along the clock beam path.

\section{Sideband thermometry on the clock transition}

We perform sideband thermometry on the clock transition (Fig. 7) using the same beam used to interrogate the atoms for clock operation. Using a standard technique of taking the ratio of the integrated area under the first red and blue sidebands [53], we obtain $\bar{n} \approx 0.66$ along the direction of the interrogation beam, oriented along one of the tight radial axes of our tweezers. From the sideband separation, we measure a trap frequency of $\omega \approx 2 \pi \times 24.5 \mathrm{kHz}$. These values are measured after cooling on the narrow ${ }^{1} \mathrm{~S}_{0} \leftrightarrow{ }^{3} \mathrm{P}_{1}$ transition for $10 \mathrm{~ms}$ [25] in a trap of depth $2447(306) E_{r}$ and adiabatically ramping down to our clock interrogation depth of 245(31) $E_{r}$.

We note that the clock transition is sufficiently narrow to observe sub-kHz inhomogeneities of trap frequencies 


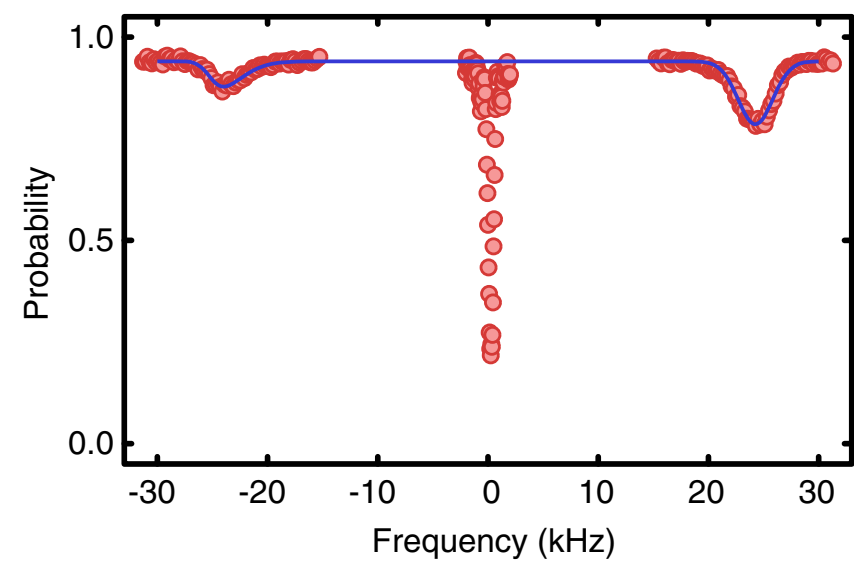

FIG. 7. Clock sideband thermometry. Array-averaged radial sideband spectrum of the optical clock transition, taken with a carrier Rabi frequency of approximately $360 \mathrm{~Hz}$. A narrow carrier stands in between two broader sidebands on the red and blue detuned sides. Sideband broadening is due mainly to small inhomogeneities in the array. A suppressed red sideband indicates significant motional ground-state population. The solid line is a simultaneous fit to two skewed Gaussians. From the ratio of the area under the red sideband to that under the blue sideband, we obtain $\bar{n} \approx 0.66$. The carrier is probed for an interrogation time of $1.4 \mathrm{~ms}$, while the sidebands are probed for $3.3 \mathrm{~ms}$.

between tweezers. This precision afforded by the clock transition allows for detailed knowledge about inhomogeneities in the array, and we envision using it for fine corrections and uniformization of an array in the future. However, for the purpose of thermometry, we broaden the clock line to a degree that these inhomogeneities are unresolved on an array-averaged level, so we may obtain a spectrum that can be easily fit and integrated. Specifically, we use a much higher magnetic field of approximately $75 \mathrm{mT}$ to obtain a carrier Rabi frequency of approximately $360 \mathrm{~Hz}$ at the same optical intensity.

\section{Evaluating Allan deviations}

Repeated interrogation introduces a bimodal distribution in the time between feedback events due to the periodic refilling of the array. To account for this variation, we approximate that all feedbacks are equally spaced in time with $\Delta t \approx 835 \mathrm{~ms}$. This method introduces a slight error $\Delta \tau \approx 100 \mathrm{~ms}$ for all $\tau$, though this error is inconsequential for fitting the long-time Allan deviation behavior. We fit all Allan deviations from $\tau=10 \mathrm{~s}$ to $\tau=100 \mathrm{~s}$, using $\sigma_{y}=A / \sqrt{\tau}$, with free parameter $A=\sigma_{y}(\tau=1 \mathrm{~s})$.

\section{APPENDIX C: STATISTICAL PROPERTIES OF THE ERROR SIGNAL}

\section{Probability distribution function}

In the absence of additional noise and given $N_{A}$ atoms, the probability of finding $N_{g}$ atoms in the ground state after a single-clock interrogation block is given by the binomial distribution $P_{\mathcal{B}}\left(N_{g} ; N_{A}, p\right)$, where $p$ is the probability of detecting an atom in its ground state following clock interrogation. The probability of measuring a given error signal $\bar{e}=\Delta N_{g} / N_{A}$ is thus given by the probability of measuring the difference in atom number $\Delta N_{g}=N_{g}^{A}-N_{g}^{B}$, where $N_{g}^{A}\left(N_{g}^{B}\right)$ is the number of atoms detected in the ground state after the $A(B)$ interrogation blocks. It can be shown that the probability distribution for $\Delta N_{g}$ is given by the convolution of two binomial distributions, $P_{*}\left(\Delta N_{g}\right.$; $\left.N_{A}, p_{A}, p_{B}\right)=\sum_{N} P_{\mathcal{B}}\left(N ; N_{A}, p_{B}\right) P_{\mathcal{B}}\left(N-\Delta N_{g} ; N_{A}, p_{A}\right)$. This discrete distribution has support on $\left\{-N_{A}\right.$, $\left.-N_{A}+1, \ldots, N_{A}\right\}$, with $2 N_{A}+1$ nonzero values. Thus, the probability distribution for $\bar{e}$ is given by $P\left(\bar{e} ; N_{A}, p_{A}, p_{B}\right)=P_{*}\left(\bar{e} N_{A} ; N_{A}, p_{A}, p_{B}\right)$. In the absence of statistical correlation between the $A$ and $B$ interrogation blocks, this distribution has a mean $\mu_{\bar{e}}=\left(p_{B}-p_{A}\right)$ and a variance $\sigma_{\bar{e}}^{2}=\left(p_{A}\left(1-p_{A}\right)+p_{B}\left(1-p_{B}\right)\right) / N_{A}$.

\section{Additional noise}

In the presence of noise, such as laser noise or finite temperature, the excitation probability $p_{A}$ and $p_{B}$ fluctuates from repetition to repetition. These fluctuations can be accounted for by introducing a joint probability density function $\pi\left(p_{A}, p_{B}\right)$, so that

$$
\begin{aligned}
P\left(\bar{e} ; N_{A}\right) & =\int d p_{A} d p_{B}\left(\pi\left(p_{A}, p_{B}\right) P\left(\bar{e} ; N_{A}, p_{A}, p_{B}\right)\right) \\
& =\left\langle P\left(\bar{e} ; N_{A}, p_{A}, p_{B}\right)\right\rangle,
\end{aligned}
$$

where $\langle\cdot\rangle$ denotes statistical averaging over $\pi\left(p_{A}, p_{B}\right)$. Assuming the mean of $P\left(\bar{e}, N_{A}\right)$ to be zero, which is equivalent to $\left\langle p_{A}\right\rangle=\left\langle p_{B}\right\rangle \equiv\langle p\rangle$, and the variance of $p_{A}$ and $p_{B}$ to be equal, $\sigma_{p_{A}}^{2}=\sigma_{p_{B}}^{2} \equiv \sigma_{p}^{2}$, it can be shown that the variance of $P\left(\bar{e}, N_{A}\right)$ is given by

$$
\sigma_{\bar{e}}^{2}=2\left(\langle p\rangle(1-\langle p\rangle)-\sigma_{p}^{2}\right) / N_{A}+2\left(\sigma_{p}^{2}-C\right),
$$

where $C$ is a correlation function between $p_{A}$ and $p_{B}$ defined as $C=\left\langle p_{A} p_{B}\right\rangle-\left\langle p_{A}\right\rangle\left\langle p_{B}\right\rangle$.

\section{Experimental data}

We can directly extract the correlation function $C$ through the results of images (2) and (4) for valid tweezers [Fig. 1(e)]. We explicitly confirm that $C$ is independent of the number of atoms used per $A B$ interrogation cycle and extract $C=-0.025$. The anticorrelation is an indication of laser noise. Note that, in contrast to $C, \sigma_{p}^{2}$ is not directly experimentally accessible as it is masked by QPN. The fit to the variance of the error signal [Fig. 2(d)] yields $\sigma_{\bar{e}}^{2}=0.379 / N_{A}+0.169$. We can thus use the fitted offset of 0.169 combined with the knowledge of $C$ to extract $\sigma_{p}^{2}=0.059$. We can alternatively use the fitted 
coefficient of the $1 / N_{A}$ term of 0.379 with the measured $\langle p\rangle=0.41$ to extract $\sigma_{p}^{2}=0.052$. To determine the contribution from QPN versus other noise sources in the standard deviation of the error signal, we take $\sigma_{\bar{e}, \mathrm{QPN}}=$ $\sqrt{2\langle p\rangle(1-\langle p\rangle) / N_{A}} / \sigma_{\bar{e}}$, which for $N_{A}=40.5$ yields $\sigma_{\bar{e}, \mathrm{QPN}}=0.26$, as quoted in the main text.

\section{APPENDIX D: EXPLOITING SINGLE-SITE-RESOLVED SIGNALS}

\section{Atom-number-dependent stability}

To study the performance of our clock as a function of atom number, we can choose to use only part of our full array for clock operation [Fig. 4(b)]. We preferentially choose atoms near the center of the array to minimize errors due to gradients in the array, e.g., from the AOD. Because of the stochastic nature of array filling, we generally use different tweezers during each filling cycle such as to always compute a signal from a fixed number of atoms. When we target a large number of atoms, some repetitions have an atom number lower than the target due to the stochastic nature of array filling, resulting in a mean atom number slightly smaller than the target, as well as a small fluctuation in atom number. The data points in Fig. 4(b) show the mean atom numbers used for clock operation, with error bars around these means (denoting the standard deviation of atom number) being smaller than the marker size.

\section{Clock comparison between two halves of the array}

We use the ability to lock to a subset of occupied traps to perform stability analysis that is sensitive to slow drifts of gradients across the array (such as from external fields or spatial variations in trap homogeneity). In this case, we lock $f_{1}$ to traps 1-40 and lock $f_{2}$ to traps $42-81$, such that noise sources that vary across the array will show a divergence in the Allan deviation at long enough times. As shown in Fig. 8, we perform this analysis for times approaching $\tau=10^{4} \mathrm{~s}$ and down to the $\sigma_{y}=1 \times 10^{-16}$ level, and we observe no violation of the $\sigma_{y} \propto 1 / \sqrt{\tau}$ behavior. Thus, we conclude that such temporal variations in gradients are not a resolvable systematic for our current experiment. However, this analysis will prove useful when using an upgraded system for which stability at the $\sigma_{y}=10^{-17}$ level or lower becomes problematic. In principle, the lock could be done on a single trap position at a time, which would allow trapby-trap systematics to be analyzed.

\section{In situ error correction}

Single-site resolution offers the opportunity both to analyze single-atom signals, as discussed in the main text, and to modify such signals before using them for feedback. As an example, the AOD introduces a spatial gradient in

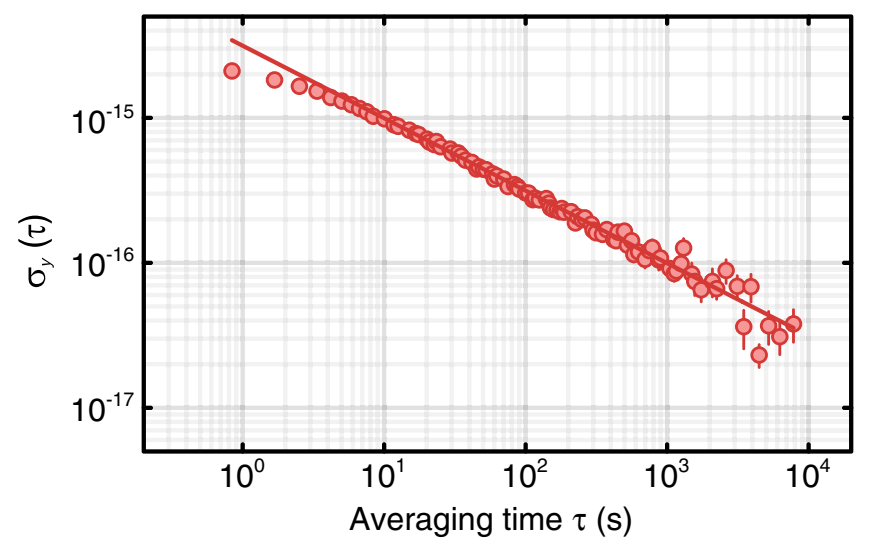

FIG. 8. Spatially resolved clock comparison. The fractional Allan deviation from an asynchronous clock comparison between the left and right halves of our array. Fitting a $1 / \sqrt{\tau}$ behavior past an initial lock onset time, we find $3.1 \times 10^{-15} / \sqrt{\tau}$, slightly higher than the result measured for a self-comparison of the full array (Fig. 4). Importantly, we see no upturn for times approaching $10^{4} \mathrm{~s}$ and below the $10^{-16}$ level, indicating that slowly varying drifts of gradients across the array do not contribute to instability up to our sensitivity.

trap frequencies across the array, leading to a spatial variation in zero crossings of the error signal [as shown in Fig. 2(b)] and subsequently leading to an increase in the Allan deviation at the $\sigma_{y} \approx 10^{-17}$ level due to stochastic trap loading. While this effect is not currently significant in our experiment, it and other array inhomogeneities may be visible to future experiments with increased stability.

Therefore, we propose that this problem can be corrected (for inhomogeneities within the probe bandwidth) by adjusting the error signal $e_{j}$ of each tweezer by a correction factor before calculating the array-averaged $\bar{e}$ that will produce feedback for the local oscillator. For instance, consider the modification $\bar{e}_{f}=\left(1 / N_{A}\right) \sum_{j} \zeta_{j} e_{j}-f_{0, j}$, where $\bar{e}_{f}$ is the tweezer-averaged error in $\mathrm{Hz}, \zeta_{j}$ is a tweezer-resolved conversion factor such as could be obtained from Fig. 2(a), and $f_{0, j}$ is the tweezer-resolved zero crossing of the error signal. This new formulation mitigates inhomogeneity without any physical change to the array. While physically enforcing array uniformity is ideal, this tool can simplify the complexity of correcting experimental systematics.

\section{APPENDIX E: TWEEZER-INDUCED LIGHT SHIFTS}

Several previous studies have analyzed the polarizability and hyperpolarizability of alkaline-earth-like atoms, including ${ }^{88} \mathrm{Sr}$, in magic-wavelength optical lattices [30-32,54]. In their analyses, these studies include the effect of finite atom temperature by Taylor expanding the lattice potential in powers of $\sqrt{I}$ ( $I$ is the lattice intensity) in the vicinity of the magic wavelength [54]. We repeat this 
TABLE I. Light shifts of a ${ }^{88} \mathrm{Sr}$ clock. The fits and predictions based on Eq. (E1) use the following values from previous studies.

\begin{tabular}{|c|c|c|c|c|}
\hline Quantity & Symbol & Unit & Value & Reference \\
\hline Magic trapping frequency & $\nu_{T}$ & $\mathrm{MHz}$ & $368554732(11)$ & [31] \\
\hline Hyperpolarizability difference & $(1 / h) \tilde{\beta}$ & $\mu \mathrm{Hz}$ & $0.45(10)$ & [55] \\
\hline Slope of $\tilde{\alpha}^{E 1}$ & $(1 / h)\left[\left(\partial \tilde{\alpha}^{E 1}\right) /(\partial \nu)\right]$ & & $19.3 \times 10^{-12}$ & [31] \\
\hline Electric dipole polarizability & $\tilde{\alpha}^{E 1}$ & $\mathrm{kHz} /\left(\mathrm{kW} / \mathrm{cm}^{2}\right)$ & $46.5976(13)$ & [56] \\
\hline Differential electric quadrupole and magnetic dipole polarizabilities & $(1 / h) \tilde{\alpha}^{q m}$ & $\mathrm{mHz}$ & $0.0(3)$ & [57] \\
\hline
\end{tabular}

derivation for an optical tweezer instead of an optical lattice.

The Gaussian tweezer intensity (assumed to have azimuthal symmetry) is given by $I(\rho, z)=I_{0}\left(w_{0} / w(z)\right)^{2} \times$ $e^{-2 \rho^{2} / w(z)^{2}}$, where $w_{0}$ is the beam waist, $I_{0}=2 P_{0} / \pi w_{0}^{2}$ is the maximum intensity, $P_{0}$ is the beam power, $w(z)=$ $w_{0} \sqrt{1+\left(z / z_{R}\right)^{2}}$, and $z_{R}=\pi w_{0}^{2} / \lambda_{T}$ is the Rayleigh range. The trapping potential is determined from this intensity $I(\rho, z)$ by the electric dipole polarizability $\alpha^{E 1}$, the electric quadrupole and magnetic dipole polarizabilities $\alpha^{q m}=$ $\alpha^{E 2}+\alpha^{M 1}$, and the hyperpolarizability effect $\beta I^{2}$.

By considering a harmonic approximation in the $x$ and $y$ directions as well as harmonic and anharmonic terms in the $z$ direction, we arrive at the following expression for the differential light shift of the clock transition in an optical tweezer, where $\rho=\sqrt{x^{2}+y^{2}}$ and $n_{\rho}\left(=n_{x}+n_{y}\right)$ and $n_{z}$ are vibrational quantum numbers along the radial and axial directions, respectively:

$$
\begin{aligned}
h \nu_{L S}= & -\left[\left(\frac{\partial}{\partial \nu} \tilde{\alpha}^{E 1}\right) \delta_{L}+\left(\frac{w_{0}}{z_{R}}\right)^{2}\left(n_{\rho}+\frac{1}{2}\right)^{2} \tilde{\beta}+\sqrt{2}\left(\frac{w_{0}}{z_{R}}\right)^{3}\left(n_{z}+\frac{1}{2}\right)\left(n_{\rho}+\frac{1}{2}\right) \tilde{\beta}+\frac{3}{8}\left(\frac{w_{0}}{z_{R}}\right)^{4}\left(n_{z}^{2}+n_{z}+\frac{1}{2}\right) \tilde{\beta}\right] u \\
& +\left[2 \sqrt{2}\left(\frac{w_{0}}{z_{R}}\right)\left(n_{\rho}+\frac{1}{2}\right)+\left(\frac{w_{0}}{z_{R}}\right)^{2}\left(n_{z}+\frac{1}{2}\right)\right] \times\left[\left(\frac{\partial}{\partial \nu} \tilde{\alpha}^{E 1}\right) \delta_{L}+\tilde{\alpha}^{q m}\right] u^{1 / 2} \\
& +\left[2 \sqrt{2}\left(\frac{w_{0}}{z_{R}}\right)\left(n_{\rho}+\frac{1}{2}\right)+\left(\frac{w_{0}}{z_{R}}\right)^{2}\left(n_{z}+\frac{1}{2}\right)\right] \tilde{\beta} u^{3 / 2}-\tilde{\beta} u^{2},
\end{aligned}
$$

where $\tilde{\alpha}^{E 1}=\Delta \alpha^{E 1}\left(E_{R} / \alpha^{E 1}\right), \quad \Delta \alpha^{E 1}=\alpha_{e}^{E 1}-\alpha_{q}^{E 1}$ is the differential $E 1$ polarizability; $\tilde{\alpha}^{q m}=\Delta \alpha^{q m}\left(E_{R} / \alpha^{E 1}\right)$, where $\Delta \alpha^{q m}$ is the differential $E 2$ and $M 1$ polarizability; $\tilde{\beta}=$ $\Delta \beta\left(E_{R} / \alpha^{E 1}\right)^{2}$, where $\Delta \beta$ is the differential hyperpolarizability; and $u=I /\left(E_{R} / \alpha^{E 1}\right)$ is the tweezer depth.

We use this formula to predict the light shifts studied in the main text (Fig. 3). As we find the results to be mostly insensitive to temperature for low temperatures, we assume zero temperature for simplicity. We allow a single fit parameter, which is an overall frequency shift due to uncertainty in the optical frequency of the trapping light. The other factors are taken from previous studies, as summarized in Table I.

[1] A. D. Ludlow, M. M. Boyd, J. Ye, E. Peik, and P. O. Schmidt, Optical Atomic Clocks, Rev. Mod. Phys. 87, 637 (2015).

[2] W. F. McGrew, X. Zhang, R. J. Fasano, S. A. Schäffer, K. Beloy, D. Nicolodi, R. C. Brown, N. Hinkley, G. Milani, M. Schioppo, T. H. Yoon, and A. D. Ludlow, Atomic Clock Performance Enabling Geodesy below the Centimetre Level, Nature (London) 564, 87 (2018).

[3] S. M. Brewer, J.-S. Chen, A. M. Hankin, E. R. Clements, C. W. Chou, D. J. Wineland, D. B. Hume, and D. R.
Leibrandt, ${ }^{27} \mathrm{Al}^{+}$Quantum-Logic Clock with a Systematic Uncertainty below 10 ${ }^{-18}$, Phys. Rev. Lett. 123, 033201 (2019).

[4] E. Oelker, R. B. Hutson, C. J. Kennedy, L. Sonderhouse, T. Bothwell, A. Goban, D. Kedar, C. Sanner, J. M. Robinson, G. E. Marti, D. G. Matei, T. Legero, M. Giunta, R. Holzwarth, F. Riehle, U. Sterr, and J. Ye, Demonstration of $4.8 \times 10^{-17}$ Stability at 1 s for Two Independent Optical Clocks, Nat. Photonics 13714 (2019).

[5] J. Grotti et al., Geodesy and Metrology with a Transportable Optical Clock, Nat. Phys. 14, 437 (2018).

[6] S. Blatt, A. D. Ludlow, G. K. Campbell, J. W. Thomsen, T. Zelevinsky, M. M. Boyd, J. Ye, X. Baillard, M. Fouché, R. Le Targat, A. Brusch, P. Lemonde, M. Takamoto, F.-L. Hong, H. Katori, and V. V. Flambaum, New Limits on Coupling of Fundamental Constants to Gravity Using ${ }^{87}$ Sr Optical Lattice Clocks, Phys. Rev. Lett. 100, 140801 (2008).

[7] T. Pruttivarasin, M. Ramm, S. G. Porsev, I. I. Tupitsyn, M. S. Safronova, M. A. Hohensee, and H. Häffner, Michelson Morley Analogue for Electrons Using Trapped Ions to Test Lorentz Symmetry, Nature (London) 517, 592 (2015).

[8] F. Scazza, C. Hofrichter, M. Höfer, P. C. De Groot, I. Bloch, and S. Fölling, Observation of Two-Orbital Spin-Exchange Interactions with Ultracold SU(N)-Symmetric Fermions, Nat. Phys. 10, 779 (2014). 
[9] W. F. McGrew, X. Zhang, H. Leopardi, R. J. Fasano, D. Nicolodi, K. Beloy, J. Yao, J. A. Sherman, S. A. Schäffer, J. Savory, R. C. Brown, S. Römisch, C. W. Oates, T. E. Parker, T. M. Fortier, and A. D. Ludlow, Towards the Optical Second: Verifying Optical Clocks at the SI Limit, Optica 6, 448 (2019).

[10] K. Kim, M.-S. Chang, S. Korenblit, R. Islam, E. E. Edwards, J. K. Freericks, G.-D. Lin, L.-M. Duan, and C. Monroe, Quantum Simulation of Frustrated Ising Spins with Trapped Ions, Nature (London) 465, 590 (2010).

[11] C. Gross and I. Bloch, Quantum Simulations with Ultracold Atoms in Optical Lattices, Science 357, 995 (2017).

[12] H. Bernien, S. Schwartz, A. Keesling, H. Levine, A. Omran, H. Pichler, S. Choi, A. S. Zibrov, M. Endres, M. Greiner, V. Vuletić, and M. D. Lukin, Probing Many-Body Dynamics on a 51-Atom Quantum Simulator, Nature (London) 551, 579 (2017).

[13] V. Lienhard, S. de Léséleuc, D. Barredo, T. Lahaye, A. Browaeys, M. Schuler, L.-P. Henry, and A. M. Läuchli, Observing the Space- and Time-Dependent Growth of Correlations in Dynamically Tuned Synthetic Ising Models with Antiferromagnetic Interactions, Phys. Rev. X 8, 021070 (2018).

[14] V. D. Ovsiannikov, A. Derevianko, and K. Gibble, Rydberg Spectroscopy in an Optical Lattice: Blackbody Thermometry for Atomic Clocks, Phys. Rev. Lett. 107, 093003 (2011).

[15] L. I. R. Gil, R. Mukherjee, E. M. Bridge, M. P. A. Jones, and T. Pohl, Spin Squeezing in a Rydberg Lattice Clock, Phys. Rev. Lett. 112, 103601 (2014).

[16] B. Braverman, A. Kawasaki, E. Pedrozo-Peñafiel, S. Colombo, C. Shu, Z. Li, E. Mendez, M. Yamoah, L. Salvi, D. Akamatsu, Y. Xiao, and V. Vuletić, Near-Unitary Spin Squeezing in ${ }^{171} \mathrm{Yb}$, Phys. Rev. Lett. 122, 223203 (2019).

[17] R. Kaubruegger, P. Silvi, C. Kokail, R. van Bijnen, A. M. Rey, J. Ye, A. M. Kaufman, and P. Zoller, Variational SpinSqueezing Algorithms on Programmable Quantum Sensors, arXiv:1908.08343 [Phys. Rev. Lett. (to be published)].

[18] B. Koczor, S. Endo, T. Jones, Y. Matsuzaki, and S. C. Benjamin, Variational-State Quantum Metrology, arXiv: 1908.08904.

[19] P. Kómár, E. M. Kessler, M. Bishof, L. Jiang, A. S. Sørensen, J. Ye, and M. D. Lukin, A Quantum Network of Clocks, Nat. Phys. 10, 582 (2014).

[20] A. J. Daley, M. M. Boyd, J. Ye, and P. Zoller, Quantum Computing with Alkaline-Earth-Metal Atoms, Phys. Rev. Lett. 101, 170504 (2008).

[21] G. Pagano, F. Scazza, and M. F. Feig, Fast and Scalable Quantum Information Processing with Two Electron Atoms in Optical Tweezer Arrays, Advanced Quantum Technologies 2, 1800067 (2019).

[22] J. P. Covey, A. Sipahigil, S. Szoke, N. Sinclair, M. Endres, and O. Painter, Telecom-Band Quantum Optics with Ytterbium Atoms and Silicon Nanophotonics, Phys. Rev. Applied 11, 034044 (2019).

[23] N. Huntemann, M. Okhapkin, B. Lipphardt, S. Weyers, C. Tamm, and E. Peik, High-Accuracy Optical Clock Based on the Octupole Transition in ${ }^{171} \mathrm{Yb}^{+}$, Phys. Rev. Lett. 108, 090801 (2012).
[24] T. R. Tan, R. Kaewuam, K. J. Arnold, S. R. Chanu, Z. Zhang, M.S. Safronova, and M. D. Barrett, Suppressing Inhomogeneous Broadening in a Lutetium Multi-ion Optical Clock, Phys. Rev. Lett. 123, 063201 (2019).

[25] J. P. Covey, I. S. Madjarov, A. Cooper, and M. Endres, 2000Times Repeated Imaging of Strontium Atoms in Clock-Magic Tweezer Arrays, Phys. Rev. Lett. 122, 173201 (2019).

[26] A. Taichenachev, V. Yudin, C. Oates, C. Hoyt, Z. Barber, and L. Hollberg, Magnetic Field-Induced Spectroscopy of Forbidden Optical Transitions with Application to LatticeBased Optical Atomic Clocks, Phys. Rev. Lett. 96, 083001 (2006).

[27] T. Akatsuka, M. Takamoto, and H. Katori, ThreeDimensional Optical Lattice Clock with Bosonic ${ }^{88} \mathrm{Sr}$ Atoms, Phys. Rev. A 81, 023402 (2010).

[28] T. L. Nicholson, S. L. Campbell, R. B. Hutson, G. E. Marti, B. J. Bloom, R. L. McNally, W. Zhang, M. D. Barrett, M. S. Safronova, G. F. Strouse, W. L. Tew, and J. Ye, Systematic Evaluation of an Atomic Clock at 21018 Total Uncertainty, Nat. Commun. 6, 6896 (2015).

[29] A. Al-Masoudi, S. Dörscher, S. Häfner, U. Sterr, and C. Lisdat, Noise and Instability of an Optical Lattice Clock, Phys. Rev. A 92, 063814 (2015).

[30] R. C. Brown, N. B. Phillips, K. Beloy, W. F. McGrew, M. Schioppo, R. J. Fasano, G. Milani, X. Zhang, N. Hinkley, H. Leopardi, T. H. Yoon, D. Nicolodi, T. M. Fortier, and A. D. Ludlow, Hyperpolarizability and Operational Magic Wavelength in an Optical Lattice Clock, Phys. Rev. Lett. 119, 253001 (2017).

[31] S. Origlia, M. S. Pramod, S. Schiller, Y. Singh, K. Bongs, R. Schwarz, A. Al-Masoudi, S. Dörscher, S. Herbers, S. Häfner, U. Sterr, and C. Lisdat, Towards an Optical Clock for Space: Compact, High-Performance Optical Lattice Clock Based on Bosonic Atoms, Phys. Rev. A 98, 053443 (2018).

[32] N. Nemitz, A. A. Jørgensen, R. Yanagimoto, F. Bregolin, and H. Katori, Modeling Light Shifts in Optical Lattice Clocks, Phys. Rev. A 99, 033424 (2019).

[33] I. D. Leroux, N. Scharnhorst, S. Hannig, J. Kramer, L. Pelzer, M. Stepanova, and P. O. Schmidt, On-Line Estimation of Local Oscillator Noise and Optimisation of Servo Parameters in Atomic Clocks, Metrologia 54, 307 (2017).

[34] S. B. Koller, J. Grotti, St. Vogt, A. Al-Masoudi, S. Dörscher, S. Häfner, U. Sterr, and C. Lisdat, Transportable Optical Lattice Clock with $7 \times 10^{-17}$ Uncertainty, Phys. Rev. Lett. 118, 073601 (2017).

[35] M. A. Norcia, A. W. Young, W. J. Eckner, E. Oelker, J. Ye, and A. M. Kaufman, Seconds-Scale Coherence on an Optical Clock Transition in a Tweezer Array, Science 366, 93 (2019).

[36] G. J. Dick, Local Oscillator Induced Instabilities in Trapped Ion Frequency Standards, Proceedings of the 19th Annual Precise Time and Time Interval Systems and Applications Meeting (1987), pp. 133-147, https://www.ion.org/ publications/abstract.cfm?articleID $=15462$.

[37] S. L. Campbell, R. B. Hutson, G. E. Marti, A. Goban, N. D. Oppong, R. L. McNally, L. Sonderhouse, J. M. Robinson, W. Zhang, B. J. Bloom, and J. Ye, A Fermi-Degenerate ThreeDimensional Optical Lattice Clock, Science 358, 90 (2017).

[38] M. Schioppo, R. C. Brown, W. F. McGrew, N. Hinkley, R. J. Fasano, K. Beloy, T. H. Yoon, G. Milani, D. Nicolodi, 
J. A. Sherman, N. B. Phillips, C. W. Oates, and A. D. Ludlow, Ultrastable Optical Clock with Two Cold-Atom Ensembles, Nat. Photonics 11, 48 (2017).

[39] M. D. Swallows, M. Bishof, Y. Lin, S. Blatt, M. J. Martin, A. M. Rey, and J. Ye, Suppression of Collisional Shifts in a Strongly Interacting Lattice Clock, Science 331, 1043 (2011).

[40] D. E. Chang, J. Ye, and M. D. Lukin, Controlling DipoleDipole Frequency Shifts in a Lattice-Based Optical Atomic Clock, Phys. Rev. A 69, 023810 (2004).

[41] R. B. Hutson, A. Goban, G. E. Marti, L. Sonderhouse, C. Sanner, and J. Ye, Engineering Quantum States of Matter for Atomic Clocks in Shallow Optical Lattices, Phys. Rev. Lett. 123, 123401 (2019).

[42] A. Cooper, J. P. Covey, I. S. Madjarov, S. G. Porsev, M. S. Safronova, and M. Endres, Alkaline-Earth Atoms in Optical Tweezers, Phys. Rev. X 8, 041055 (2018).

[43] F. Nogrette, H. Labuhn, S. Ravets, D. Barredo, L. Béguin, A. Vernier, T. Lahaye, and A. Browaeys, Single-Atom Trapping in Holographic 2D Arrays of Microtraps with Arbitrary Geometries, Phys. Rev. X 4, 021034 (2014).

[44] F. Riehle, Frequency Standards (Wiley, New York, 2003).

[45] M. A. Norcia, A. W. Young, and A. M. Kaufman, Microscopic Control and Detection of Ultracold Strontium in Optical-Tweezer Arrays, Phys. Rev. X 8, 041054 (2018).

[46] M. A. Norcia, Coupling Atoms to Cavities with Narrow Linewidth Optical Transitions: Applications to Frequency Metrology, arXiv:1908.11442.

[47] D. J. Wineland and W. M. Itano, Laser Cooling of Atoms, Phys. Rev. A 20, 1521 (1979).

[48] S. de Léséleuc, D. Barredo, V. Lienhard, A. Browaeys, and T. Lahaye, Analysis of Imperfections in the Coherent Optical Excitation of Single Atoms to Rydberg States, Phys. Rev. A 97, 053803 (2018).
[49] K. Numata, A. Kemery, and J. Camp, Thermal-Noise Limit in the Frequency Stabilization of Lasers with Rigid Cavities, Phys. Rev. Lett. 93, 250602 (2004).

[50] Y. Y. Jiang, A. D. Ludlow, N. D. Lemke, R. W. Fox, J. A. Sherman, L.-S. Ma, and C. W. Oates, Making Optical Atomic Clocks More Stable with $10^{-16}$-Level Laser Stabilization, Nat. Photonics 5, 158 (2011).

[51] S. Webster and P. Gill, Force-Insensitive Optical Cavity, Opt. Lett. 36, 3572 (2011).

[52] Z. Barber, C. Hoyt, C. Oates, L. Hollberg, A. Taichenachev, and V. Yudin, Direct Excitation of the Forbidden Clock Transition in Neutral ${ }^{174} \mathrm{Yb}$ Atoms Confined to an Optical Lattice, Phys. Rev. Lett. 96, 083002 (2006).

[53] C. Han, M. Zhou, X. Zhang, Q. Gao, Y. Xu, S. Li, S. Zhang, and X. Xu, Carrier Thermometry of Cold Ytterbium Atoms in an Optical Lattice Clock, Sci. Rep. 8, 7927 (2018).

[54] H. Katori, V. D. Ovsiannikov, S. I. Marmo, and V. G. Palchikov, Strategies for Reducing the Light Shift in Atomic Clocks, Phys. Rev. A 91, 052503 (2015).

[55] R. Le Targat, L. Lorini, Y. Le Coq, M. Zawada, J. Guéna, M. Abgrall, M. Gurov, P. Rosenbusch, D. G. Rovera, B. Nagórny, R. Gartman, P. G. Westergaard, M. E. Tobar, M. Lours, G. Santarelli, A. Clairon, S. Bize, P. Laurent, P. Lemonde, and J. Lodewyck, Experimental Realization of an Optical Second with Strontium Lattice Clocks, Nat. Commun. 4, 2109 (2013).

[56] T. Middelmann, S. Falke, C. Lisdat, and U. Sterr, High Accuracy Correction of Blackbody Radiation Shift in an Optical Lattice Clock, Phys. Rev. Lett. 109, 263004 (2012).

[57] P. G. Westergaard, J. Lodewyck, L. Lorini, A. Lecallier, E. A. Burt, M. Zawada, J. Millo, and P. Lemonde, LatticeInduced Frequency Shifts in Sr Optical Lattice Clocks at the 10 ${ }^{-17}$ Level, Phys. Rev. Lett. 106, 210801 (2011). 